The INL is a

U.S. Department of Energy

National Laboratory

operated by

Battelle Energy Alliance

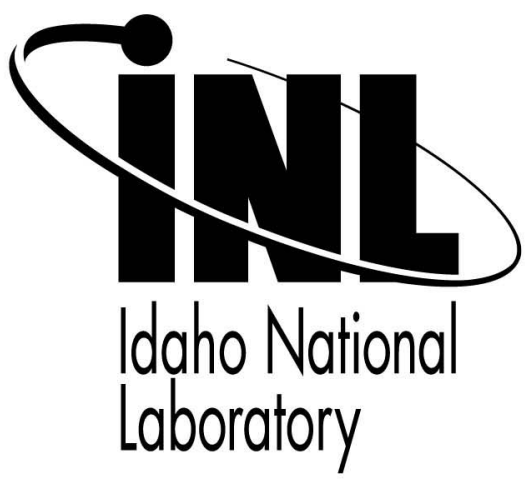

\title{
Time-Dependent Delayed Signatures from Energetic Photon Interrogations
}

\section{Conference on Accelerator Applications in Research and Industry}

\author{
Daren R. Norman \\ James L. Jones \\ Brandon W. Blackburn \\ Kevin J. Haskell \\ James T. Johnson \\ Scott M. Watson \\ Alan W. Hunt \\ Randy Spaulding \\ Frank Harmon
}

\section{August 2006}

This is a preprint of a paper intended for publication in a journal or proceedings. Since changes may be made before publication, this preprint should not be cited or reproduced without permission of the author. This document was prepared as an account of work sponsored by an agency of the United States Government. Neither the United States Government nor any agency thereof, or any of their employees, makes any warranty, expressed or implied, or assumes any legal liability or responsibility for any third party's use, or the results of such use, of any information, apparatus, product or process disclosed in this report, or represents that its use by such third party would not infringe privately owned rights. The views expressed in this paper are not necessarily those of the United States Government or the sponsoring agency. 


\title{
Time-dependent delayed signatures from energetic photon interrogations
}

\author{
Daren R. Norman ${ }^{\text {a,*, James L. Jones }}{ }^{\text {a }, ~ B r a n d o n ~ W . ~ B l a c k b u r n ~}{ }^{\text {a }}$, Kevin J. Haskell ${ }^{\text {a }}$, \\ James T. Johnson a, Scott M. Watson ${ }^{\mathrm{a}}$, Alan W. Hunt ${ }^{\mathrm{b}}$, \\ Randy Spaulding ${ }^{\mathrm{b}}$, Frank Harmon ${ }^{\mathrm{b}}$ \\ ${ }^{a}$ Idaho National Laboratory, P.O. Box 1625, Idaho Falls, ID 83415-2802, United States \\ b Idaho State University, Pocatello, ID, United States
}

\begin{abstract}
Pulsed photonuclear interrogation environments generated by $8-24 \mathrm{MeV}$ electron linac are rich with time-dependent, material-specific, radiation signatures. Nitrogen-based explosives and nuclear materials can be detected by exploiting these signatures in different delayed-time regions. Numerical and experimental results presented in this paper show the unique time and energy dependence of these signatures. It is shown that appropriate delayed-time windows are essential to acquire material-specific signatures in pulsed photonuclear assessment environments. These developments demonstrate that pulsed, high-energy, photon-inspection environments can be exploited for time-dependent, material-specific signatures through the proper operation of specialized detectors and detection methods.
\end{abstract}

Keywords: Photonuclear; Nuclear materials detection; Explosives detection; Gamma-ray spectroscopy; HPGe detector; Time-dependent signatures

\section{Introduction}

Pulsed photonuclear assessment (PPA) technologies are being developed by Idaho National Laboratory (INL) and the Idaho Accelerator Center (IAC) to address advanced cargo inspection and material identification needs [1-3]. The high-energy photons are generated by bremsstrahlung from 8 to $24 \mathrm{MeV}$ electron linacs. For most commercialoff-the-shelf (COTS) spectroscopy systems, operating in the PPA environment is difficult. A major operational problem is the recovery from the linac's photon pulse, a very high flux of photons that last for a few microseconds and the accompanying strong radio frequency (RF) fields. Earlier PPA related research and development efforts have

\footnotetext{
Corresponding author. Tel.: +1 208526 3953; fax: +1 2085265208.

E-mail address: Daren.Norman@inl.gov (D.R. Norman).
}

provided High Purity Germanium (HPGe) gamma-ray spectroscopy detection systems capable of operating in these intense environments [4].

Use of these modified HPGe detectors shows the rich, material-specific, gamma-ray signatures generated in the PPA environment. However, during previous testing, the detection sensitivity has changed drastically, with small changes in the experimental configurations. To understand this sensitivity, both numerical models and experiments were used. The results of the models and experiments indicate that there is strong time dependence relative to the interrogating photon pulse for gamma-ray signatures. An experimentally-based graphic of the time-dependent HPGe detector spectrum is shown in Fig. 1. The time axis in Fig. 1 is relative to the photon pulse from the linac, and the energy axis has key energies identified. The high-energy portion of the spectrum dies away within a few $100 \mu$ s after the pulse. This die away can be seen in the upper right side 


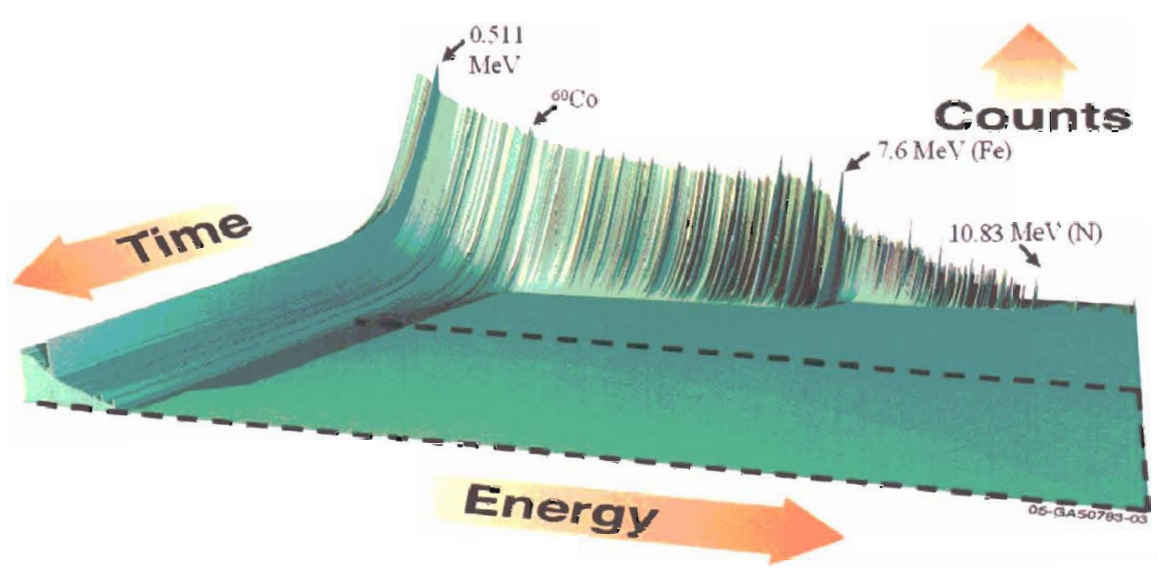

Fig. 1. Representation of time and energy dependent response of a HPGe detector in a PPA environment with ammonium nitrate-fuel oil explosive surrogate, a dashed box highlighting the long-delayed region $(4-8 \mathrm{~ms})$ used for nuclear material detection.

of Fig. 1, indicated by the lack of counts following the pulse. Soon after the pulse the spectrum is dominated by neutron capture, gamma-ray $(\mathrm{n}, \gamma)$ and scatter reactions. Therefore, to detect material-specific signatures based on the $(\mathrm{n}, \gamma)$ reactions, like nitrogen-rich explosives, counting soon after the pulse is essential. Conversely, for increased detection sensitivity for radioactive nuclei and isomers, like fission fragments, it is necessary to wait for the $(n, \gamma)$ and scatter reactions to die away. This long-delayed region is highlighted in Fig. 1 with a dashed line which is about 4 $8 \mathrm{~ms}$ after the pulse. In this delayed-time region, the highest energy material-specific signature is the ${ }^{60} \mathrm{Co}$, which was used as a constant calibration source.

\section{Neutron capture gamma-ray signatures}

Assessments using nitrogen-based explosives and the time-dependent nature of the characteristic neutron cap- ture gamma-rays ( $\mathrm{n}, \gamma$ ) emissions were evaluated. To better understand the time dependence, a numerical model was developed using MCNPX [5]. The model predicted the time dependence of the ${ }^{14} \mathrm{~N}(\mathrm{n}, \gamma)$ reactions in the ammonium nitrate-fuel oil (ANFO) surrogate and the surrounding air. Fig. 2 shows the time-dependent numerical predictions for ${ }^{14} \mathrm{~N}(\mathrm{n}, \gamma)$ reactions from the ANFO drum and surrounding air. The results of the ANFO drum show that within the first millisecond the capture reaction rate drops about five orders of magnitude. This was the first indication of strong time dependence. The numerical predictions focused the testing on quickly recovering from the photon pulse so the ${ }^{14} \mathrm{~N}(\mathrm{n}, \gamma)$ reaction gamma-rays could be detected. The model also indicates that the majority of the ${ }^{14} \mathrm{~N}(\mathrm{n}, \gamma)$ reactions before about $500 \mu$ s are from the ANFO drum and not the surrounding air. However, after about $1 \mathrm{~ms}$, the surrounding air provided more ${ }^{14} \mathrm{~N}(\mathrm{n}, \gamma)$ reactions than the ANFO drum. The configuration

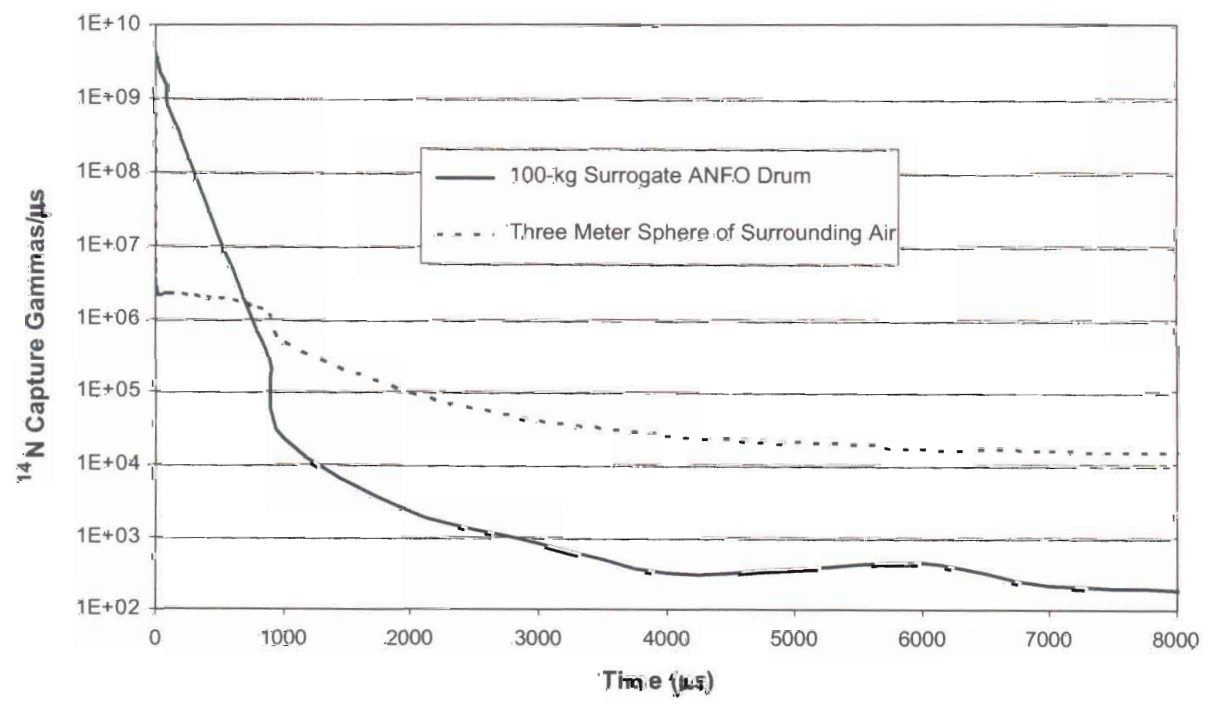

Fig. 2. Numerical predictions using MCNPX showing the time-dependent capture gamma-ray response for $100 \mathrm{~kg}$ of ANFO in a 30 -gal drum and the surrounding air. 


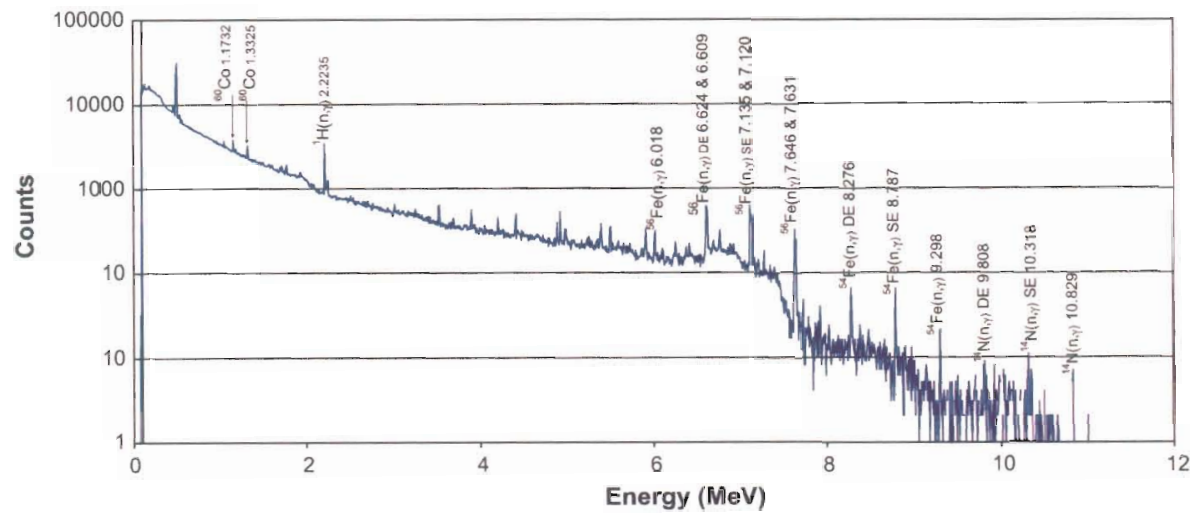

Fig. 3. HPGe spectrum of a 30-gal drum of ANFO surrogate with $10.829 \mathrm{MeV}$ nitrogen peak acquired between linac pulses.

modeled contained $100 \mathrm{~kg}$ of ANFO surrogate in a 30 -gal drum. The 30-gal drum was centered in a $20 \mathrm{MeV}$ linac beam at $3 \mathrm{~m}$ from the tungsten electron-to-photon converter, and the $3-\mathrm{m}$ sphere of air surrounding the drum.

Gamma-ray spectra of the ${ }^{14} \mathrm{~N}(\mathrm{n}, \gamma)$ reaction were collected using a modified HPGe detector. The ANFO surrogate was moved $50 \mathrm{~cm}$ from the detector, which was outside of the primary beam. However, the collected gamma-ray spectra showed the predicted time dependence. Fig. 3 highlights one of the acquired spectra with the first major peak at $10.833 \mathrm{MeV}$ [6], which is the full energy peak for the ${ }^{14} \mathrm{~N}(\mathrm{n}, \gamma)$ reaction. The full energy peak contained 12 counts, and the single escape peak contained 25 counts. Below the nitrogen peaks, in Fig. 3, is the full energy peak for iron at $9.298 \mathrm{MeV}$. The spectrum shown in Fig. 3 was collected with an INL-modified pre-amp on an $18 \%$ Princeton Gamma Tech HPGe detector. The detector was encased in $20 \mathrm{~cm}$ of borated polyethylene (B-Poly) and a $2.5 \mathrm{~cm}$ collimator, with $0.635 \mathrm{~cm}$ of lead and $20 \mathrm{~cm}$ of BPoly over the opening of the detector. The spectrum was collected for $2000 \mathrm{~s}$ with the accelerator operated at $60 \mathrm{~Hz}$ and very low beam current.

Assessments of the time-dependent nature of several spectra were evaluated with both gated windows and listmode acquisition systems. As predicted with the numerical model, the $(\mathrm{n}, \gamma)$ response is very time-dependent. Fig. 1 was developed using the time-dependent list mode counts from the HPGe detector. In this graphic the $1.333-\mathrm{MeV}$ ${ }^{60} \mathrm{Co}$ source and the $10-\mathrm{MeV}{ }^{14} \mathrm{~N}$ reactions and their time dependence are clearly visible. The linac was operating at $60 \mathrm{~Hz}$, and this data collection was for $30 \mathrm{~min}$. As both the numerical model and the experimental results show the $(n, \gamma)$ reactions in the PPA environment are extremely time-dependent.

Therefore, for effective material $(\mathrm{n}, \gamma)$ signature acquisitions, recovery within $100 \mu$ s is necessary. Small changes in testing configurations can result in detector recovery times ranging from tens to hundreds of microseconds, which makes a huge difference in the ability to detect these fast die-away signatures. However, for materials-specific signatures from radioactive nuclei and isomers such as those emitted from nuclear material fission fragments, the recovery time is not as critical, because the detection of longerlived components requires that the $(n, \gamma)$ signatures have time to die away.

\section{Nuclear material fission fragment gamma-ray signatures}

The real-time measurements of characteristic fission fragments in PPA environments have proven to be difficult. While it has been proposed by many that the delayed gamma-ray emissions above 3 or $4 \mathrm{MeV}$ could be used to indicate the presence of nuclear materials, these measurements are made after the interrogating source is turned off [7]. As Fig. 1 shows, soon after the interrogating pulse, gamma-ray energies are well in excess of $5 \mathrm{MeV}$. Therefore, simply using the energies above 3 or $4 \mathrm{MeV}$ would not work well for this PPA technique. These high-energy $(\mathrm{n}, \gamma)$ also introduce a major noise signal to the low-energy $(<5 \mathrm{MeV})$ spectroscopy needed for nuclear material fission fragment detection. However, if the HPGe detector acquisitions are delayed several milliseconds after the interrogating pulse as shown by the dashed box region in Fig. 1, it becomes possible to measure the fission fragment signatures between pulses.

An INL modified HPGe detector gated to operate from 4 to $8 \mathrm{~ms}$ was used to obtain spectra from the nuclearmaterial fission-fragments. Fig. 4 shows HPGe spectra for $300 \mathrm{~s}$ collection time (150-s active time because of gating) during linac operations with and without nuclear material. When nuclear material was present the two spectra clearly separate below about $5 \mathrm{MeV}$. By comparing these two spectra at $2 \mathrm{MeV}$, it can be seen that the counts per channel with nuclear material is an order-of-magnitude higher than without nuclear material. In Fig. 4 there are several high-energy peaks causing the divergence of these two spectra. Additionally, a well-defined peak is highlighted between the ${ }^{60} \mathrm{Co}$ calibration source peaks and the ${ }^{28} \mathrm{Al}$ activation peak at about $1.43 \mathrm{MeV}$ (channel width $6.2 \mathrm{eV}$ ). Fig. 5 shows an increased energy resolution of the energies between 1.15 and $1.8 \mathrm{MeV}$. With the increased energy resolution, the $1.43 \mathrm{MeV}$ peak shown in Fig. 4 splits 


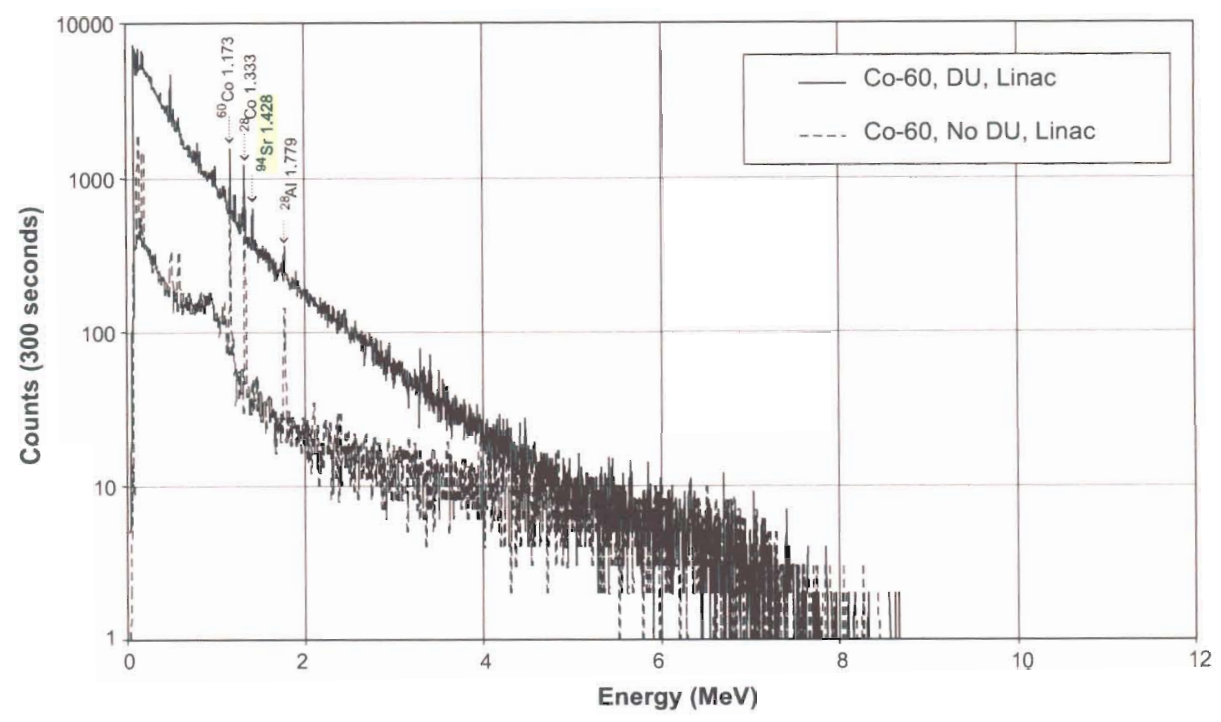

Fig. 4. Gamma-ray spectra acquired between linac pulses from HPGe detector with and without nuclear material. The ${ }^{94} \mathrm{Sr}$ peak is highlighted, and the peak is only present with nuclear material.

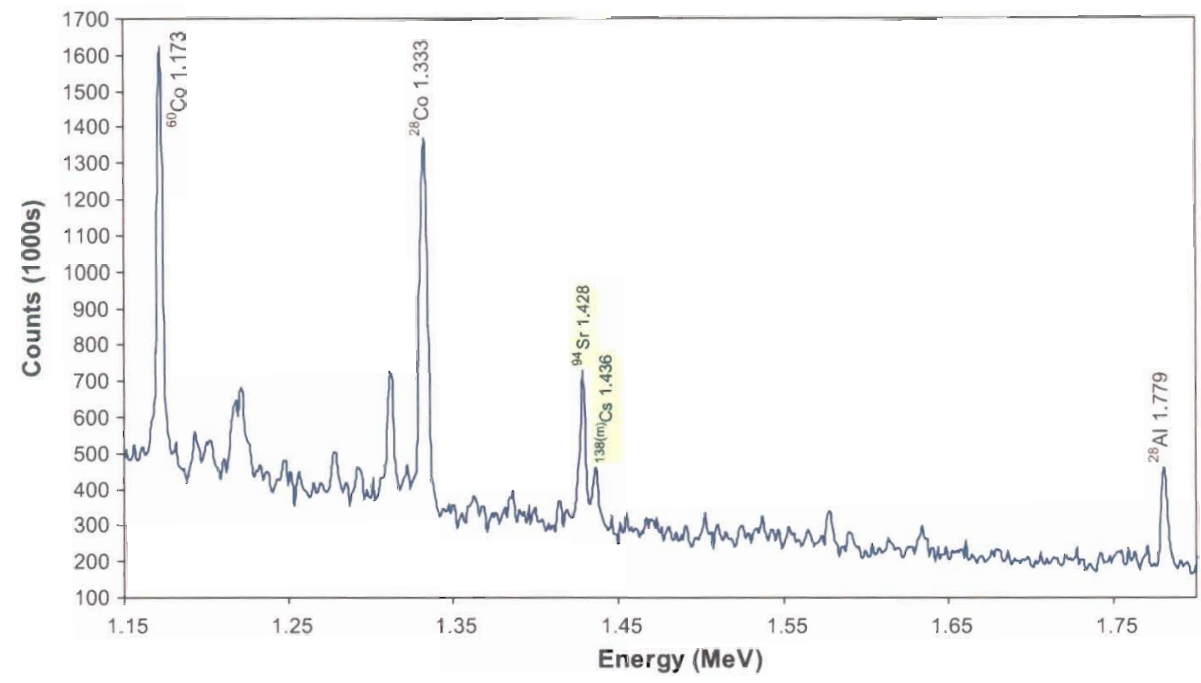

Fig. 5. Higher resolution spectrum with ${ }^{94} \mathrm{Sr}$ and ${ }^{138} \mathrm{Cs}$ fission fragments identified.

into two separate peaks; one at 1.428 and another at $1.436 \mathrm{MeV}$. The fission fragments that produced these two peaks are ${ }^{94} \mathrm{Sr}(1.4277 \mathrm{MeV}, \quad 75 \mathrm{~s} \mathrm{~T} 1 / 2), \quad{ }^{138} \mathrm{Cs}$ (1.43586 MeV, $33.41 \mathrm{~m} \mathrm{~T}_{1 / 2}$ ), and ${ }^{138 \mathrm{~m}} \mathrm{Cs}(1.4360 \mathrm{MeV}$, $2.9 \mathrm{~m} \mathrm{~T}_{1 / 2}$ ). Even in this small $650 \mathrm{keV}$ section of the spectrum after only $150 \mathrm{~s}$ acquisition many fission fragments are identifiable. The purpose of this paper is to show the rich material-specific signatures gamma-ray emissions provided by the PPA environment and their time dependence relative to the photon pulse. Therefore, a complete list of identified fission fragments is not provided.

The nuclear material experiments were conducted with the INL Varitron (2-12 MeV linac) operated at $10 \mathrm{MeV}$, $125 \mathrm{~Hz}$, and an average beam current of $3.6 \mu \mathrm{A}$. The experiment used a $1.2-\mathrm{kg}$ depleted uranium (DU) plate of nuclear material placed $1 \mathrm{~m}$ from the photon-to-electron converter, and the HPGe detector was placed $70 \mathrm{~cm}$ below the DU on beam centerline. The HPGe had $10-15 \mathrm{~cm}$ of lead collimation towards the DU and with an open back.

\section{Conclusion}

It has been demonstrated that modified HPGe detectors operated properly can detect material-specific gamma-ray signatures in PPA environments. To detect neutron capture gamma-ray signatures, fast recovery after the photon pulse is necessary. For detecting radioactive nuclei and isomers, longer delays after the photon pulse are needed. Even though PPA environments are rich with material-specific signatures, existing COTS detection systems and 
photonuclear sources have not been developed to exploit these signatures. As these detection technologics develop they could complement high-energy cargo inspection systems.

\section{References}

[1] J.L. Jones et al., Proof-of-Concept Assessment of a Photofission-Based Interrogation System for the Detection of Shielded Nuclear Material, Idaho National Laboratory Report Number INEEL/EXT-200001523,2000
[2] J.L. Jones et al., Detection of shielded nuclear material in a cargo container, Nucl. Instr. and Meth. A 562 (2006) 1085.

[3] D.R. Norman et al., Inspection applications with higher electron beam energies, Nucl. Instr. and Meth. B 241 (2005) 787.

[4] J.L. Jones et al., Detection of pulsed, Bremsstrahlung-induced, prompt neutron capture gamma-rays with a HPGe detector, SPI Proceedings 2867 (1996) 202.

[5] J.S. Hendricks et al., MCNPX, Version 2.5.e, LANL Report, LANUR-04-0569, 2004.

[6] M.A. Lone et al., Prompt gamma rays from thermal-neutron capture, Atom. Data Nucl. Data Tables 26 (6) (1981) 511.

[7] D.K. Wehe, H. Yang, M.H. Jones, Observation of U-238 photofission products, IEEE Trans. on Nucl. Sci. 53 (3) (2006). 